\title{
Imaging brain cortisol regulation in PTSD with a target for 11 $\beta$-hydroxysteroid dehydrogenase type 1
}

\author{
Shivani Bhatt, ${ }^{1}$ Ansel T. Hillmer, ${ }^{2,3,4}$ Aleksandra Rusowicz, ${ }^{3}$ Nabeel Nabulsi, ${ }^{2,4}$ David Matuskey, ${ }^{2,3,4}$ Gustavo A. Angarita, ${ }^{3,4}$ \\ Soheila Najafzadeh, ${ }^{4}$ Michael Kapinos, ${ }^{4}$ Steven M. Southwick, ${ }^{3,5}$ John H. Krystal, ${ }^{3,5}$ Richard E. Carson, ${ }^{3,4}$ Yiyun Huang, ${ }^{2,4}$ \\ and Kelly P. Cosgrove $e^{1,2,3,4,5}$
}

${ }^{1}$ Interdepartmental Neuroscience Program, Yale University, New Haven, Connecticut, USA. ${ }^{2}$ Department of Radiology and Biomedical Imaging, ${ }^{3}$ Department of Psychiatry, and ${ }^{4}$ Yale PET Center, Yale School of Medicine, New Haven, Connecticut, USA. 5US Department of Veterans Affairs, National Center for Posttraumatic Stress Disorder, Clinical Neurosciences Division, VA Connecticut Healthcare System, West Haven, Connecticut, USA

BACKGROUND. Investigations of stress dysregulation in posttraumatic stress disorder (PTSD) have focused on peripheral cortisol, but none have examined cortisol in the human brain. This study used positron emission tomography (PET) to image

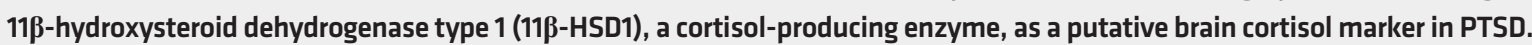

METHODS. Sixteen individuals with PTSD and 17 healthy, trauma-exposed controls (TCs) underwent PET imaging with [18 F] AS2471907, a radioligand for 11ß-HSD1.

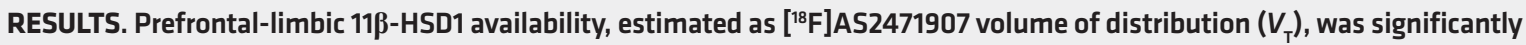
higher in the PTSD group compared with the TC group $(\beta=1.16, P=0.0057)$. Lower prefrontal-limbic 11 $\beta$-HSD1 availability was related to greater overall PTSD severity $\left(R^{2}=0.27, P=0.038\right)$ in the PTSD group. 11 -HSD1 availability was not related to plasma cortisol levels $\left(R^{2}=0.026, P=0.37\right)$. In a PTSD subset $(n=10)$, higher $11 \beta$-HSD1 availability was associated with higher availability of translocator protein (TSPO), a microglial marker $(\beta=4.40, P=0.039)$.

CONCLUSION. Higher brain cortisol-producing 11ß-HSD1 in the PTSD group may represent a resilience-promoting neuroadaptation resulting in lower PTSD symptoms. Along with preliminary associations between 11ß-HSD1 and TSPO, corroborating previous evidence of immune suppression in PTSD, these findings collectively challenge previous hypotheses of the deleterious effects of both excessive brain glucocorticoid and brain immune signaling in PTSD.

FUNDING. Brain and Behavior Research Foundation Independent Investigator Grant, National Institute of Mental Health grants F30MH116607 and R01MH110674, the Veterans Affairs National Center for PTSD, the Gustavus and Louise Pfeiffer Foundation Fellowship, Clinical and Translational Science Awards grant UL1 TR000142 from the NIH National Center for Advancing Translational Science.

\section{Introduction}

The hypothalamic-pituitary-adrenal (HPA) axis, a major neuroendocrine stress system, has been a central focus to understanding the pathophysiology of how posttraumatic stress disorder (PTSD) arises in $9.3 \%$ of persons experiencing any single traumatic event (1-9). Though studies have converged around findings of a hypocortisolemic HPA axis phenotype - lower morning plasma cortisol, lower daily urine cortisol output, and enhanced negative feedback suppression (4) - there have been several discrepancies in subsequent measurements of HPA axis activity in PTSD (10-12). Furthermore, the majority of PTSD studies have examined peripheral cortisol levels or peripheral glucocorticoid receptor (GR) sensitivity in lymphocytes in PTSD (13) as proxies for the brain cortisol sig-

Conflict of interest: The authors have declared that no conflict of interest exists. Copyright: @ 2021, American Society for Clinical Investigation.

Submitted: April 12, 2021; Accepted: August 31, 2021; Published: October 15, 2021 Reference information: J Clin Invest. 2021;131(20):e150452.

https://doi.org/10.1172/JCI150452. naling proposed to regulate the HPA axis at the corticolimbic level (14). These previous examinations of stress dysregulation in PTSD assume that the major source of glucocorticoid signaling in the brain is peripherally produced adrenal cortisol that subsequently enters the brain. However, peripheral cortisol enters the brain at a slow rate (15) and may only contribute to approximately $5 \%$ of the cortisol in the brain (16), arguing for local regulation of cortisol production in the brain as an important modulator of the HPA axis activity and fear memory processes found to be aberrant in PTSD.

$11 \beta$-hydroxysteroid dehydrogenase type 1 (11 $\beta$-HSD1) is an enzyme that intracellularly regenerates cortisol via reduction of the highly brain-permeable but inactive cortisone (17). This enzyme is estimated to contribute to at least $50 \%$ of the cortisol in the brain (18). To date, investigations of cortisol concentrations in the brain, either entering the brain from the periphery or produced in situ by 11 $\beta$-HSD1, have not been performed in individuals with PTSD. $\left[{ }^{18} \mathrm{~F}\right]$ AS2471907 is a novel radiotracer with specific binding to 11ß-HSD1 (19) that has recently been characterized in humans (20), enabling in vivo imaging of $11 \beta-H S D 1$ in PTSD. Critically, this is the only 


\section{Table 1. Participant characteristics and injection parameters}

\begin{tabular}{|c|c|c|c|}
\hline Characteristics & Control $(n=17)$ & PTSD $(n=16)$ & $P$ value \\
\hline Age, years & $33 \pm 8$ & $37 \pm 10$ & 0.15 \\
\hline Sex & 8 female, 9 male & 8 female, 8 male & 0.87 \\
\hline Body mass index & $26.1 \pm 4.7$ & $27.8 \pm 5.6$ & 0.38 \\
\hline Ethnicity, \% & & & 0.34 \\
\hline African American & $6(35)$ & $5(56)$ & \\
\hline White & $5(29)$ & $9(31)$ & \\
\hline Hispanic & $4(24)$ & $1(6)$ & \\
\hline Other & $2(12)$ & $1(6)$ & \\
\hline \multicolumn{4}{|l|}{ Clinical characteristics } \\
\hline Screening-day CAPS-5 (n) ${ }^{A}$ & $3 \pm 5(14)$ & $31 \pm 10(16)$ & $<0.001$ \\
\hline Scan-day PCL & $3 \pm 4$ & $31 \pm 16$ & $<0.001$ \\
\hline \multicolumn{4}{|l|}{ Trauma exposure(s) } \\
\hline Sexual trauma & 6 & 9 & - \\
\hline Physical trauma & 5 & 11 & - \\
\hline Childhood trauma & 12 & 15 & - \\
\hline Combat & 3 & 4 & - \\
\hline Accident/natural disaster & 6 & 8 & - \\
\hline Years since primary trauma & $11 \pm 11$ & $15 \pm 11$ & 0.28 \\
\hline Current tobacco users & 4 & 7 & 0.22 \\
\hline Current cannabis users & 2 & 5 & 0.17 \\
\hline Current alcohol users & 13 & 11 & 0.62 \\
\hline Current comorbid MDD & - & 10 & - \\
\hline Currently on psychotropic medication & - & 10 & - \\
\hline \multicolumn{4}{|l|}{ Injection parameters } \\
\hline Injected dose (MBq) & $92 \pm 18$ & $95 \pm 10$ & 0.46 \\
\hline Injected mass ( $\mu \mathrm{g} / \mathrm{kg})$ & $0.0081 \pm 0.0064$ & $0.0050 \pm 0.0029$ & \\
\hline
\end{tabular}

${ }^{A}$ Significant at $P<0.05$; assessed from 2-sided independent samples $t$ test. CAPS-5: Clinician Administered PTSD Scale from the DSM-5 to confirm PTSD diagnosis; PCL: PTSD Checklist to quantify symptom severity; MDD: major depressive disorder. Values are mean $\pm S D$, unless otherwise specified. of brain cortisol resulting from higher 11ß-HSD1 levels could be a mechanism underlying 2 phenomena observed in PTSD: first, the chronic HPA axis suppression and enhanced negative feedback, which may be regulated by brain cortisol produced in situ (22), and second, the pathology rooted in fear memory (i.e., threat symptoms, including intrusive reexperiencing, trauma-related psychological and physiological reactivity, and exaggerated startle; ref. 28).

Variability has been observed in HPA axis alterations along symptom dimensions of PTSD, such as loss (i.e. anhedonia), emotional detachment, and restricted affect (11), and in PTSD with comorbid major depressive disorder (MDD) compared with PTSD alone (4). Meanwhile different HPA axis alterations altogether have been observed in MDD (29). Assessing PTSD symptom dimensions would shed greater light on PTSD-specific associations with novel biomarkers like 11 $\beta$-HSD1 and on the heterogeneity within PTSD that has been observed across HPA axis findings. Peripheral immune modulation has also been shown to influence HPA axis alterations observed in PTSD $(30,31)$. Recently, a postmortem PTSD transcriptomic study was the first to look at both glucocorticoid and immune signaling markers centrally, identifying molecular network hubs in PTSD: FKBP5, a glucocorticoid receptor chaperone, and $U B A 7$, an enzyme in inflammatory gene expression cascade (32). The present study offered the opportunity to examine, in vivo, additional central glucocorticoid and immune markers through imaging of 11 $\beta$-HSD1 alongside a microglial marker, translocator protein (TSPO), in the same individuals with PTSD. Given recent findings of lower TSPO in PTSD that suggested neuroimmune suppression in currently viable in vivo imaging marker of brain cortisol-related signaling. Previous radiotracers targeting GRs were not suitable for brain imaging due to poor blood-brain barrier penetration, rapid metabolism, or high levels of nonspecific binding (21).

$11 \beta$-HSD1 is a putative modulator of HPA axis activity via cortisol-GR signaling in cortical and limbic areas (14), based on preclinical studies linking higher levels of the brain cortisol-producing enzyme with enhanced HPA axis suppression (22). Thus, higher

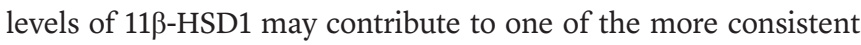
observations in PTSD: enhanced sensitivity of the HPA axis negative feedback loop. Examining 11 $\beta$-HSD1 as a mediator of brain cortisol-GR signaling may also help elucidate the apparent discrepancies between findings of heightened GR sensitivity in PTSD, on one hand (13), and increased FKBP5-GR interactions leading to GR resistance in PTSD, on the other (23). Apart from a putative role in regulating the HPA axis, most preclinical studies demonstrate that higher 11 $\beta$-HSD1 levels and corresponding increased concentrations of hippocampal corticosterone (rodent cortisol analog) appear to impair spatial memory in the context of aging or acute stress (16, 24-26).11 $\beta$-HSD1 activity may have an opposite effect on fear memory processes, with reversible 11 $\beta$-HSD1 inhibition being shown to impair fear memory consolidation (27). Increased levels contrast with the long-proposed proinflammatory pathophysiology of PTSD (33), the analysis of brain glucocorticoid and immune regulation together may further elucidate the brain stress-immune molecular network that distinguishes PTSD from $\operatorname{MDD}(33,34)$.

In the current investigation, we used positron emission tomography (PET) imaging with $\left.{ }^{[18} \mathrm{F}\right] \mathrm{AS} 2471907$ to examine brain $11 \beta$-HSD1, a putative marker of cortisol regulation, in relation to PTSD pathophysiology. To our knowledge, this study is the first to examine a putative brain cortisol marker in vivo in individuals with trauma and PTSD, and it has the potential to reveal the brain glucocorticoid mechanisms underlying the pathophysiology and diversity of symptom presentations in PTSD. Additionally, this study allowed the examination of relationships between the brains' stress and immune systems in PTSD, within-subject, in a subset of individuals who had also completed a $\left[{ }^{11} \mathrm{C}\right]$ PBR2 8 PET scan as part of a previous study examining the microglial marker TSPO in PTSD (33).

\section{Results}

Participants. Individuals with PTSD or trauma exposure but without PTSD (trauma-exposed controls, TCs) were recruited using IRB-approved advertisements. Written informed consent was 


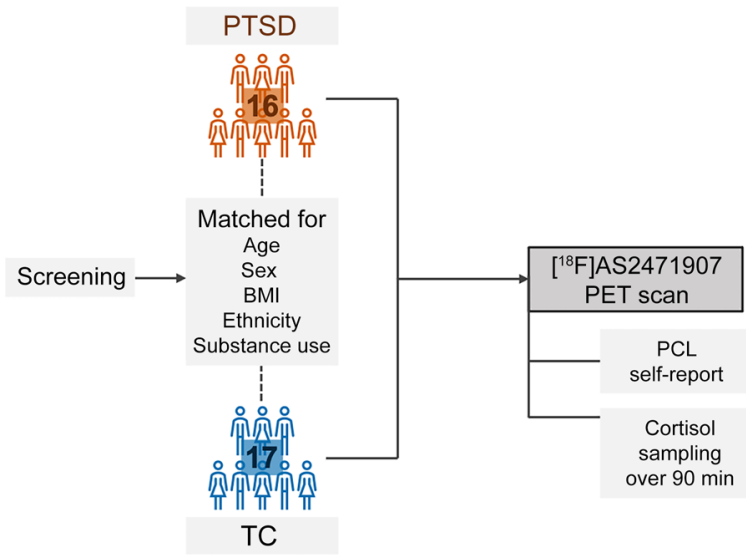

Figure 1. Schematic of study design. Participants in the PTSD group $(n=$ 16), who were screened using the CAPS-5, were matched on age, sex, BMI, ethnicity, and substance use to trauma-exposed controls (TCs, $n=17$ ). All participants underwent a $\left[{ }^{18} \mathrm{~F}\right] \mathrm{AS} 2471907 \mathrm{PET}$ scan, completed a PCL self-report on scan day, and had plasma cortisol levels measured every 30 minutes from 0 to 90 minutes after the radiotracer injection.

obtained before participation. Participants in the 2 groups were well-matched on demographic characteristics such as age, sex, BMI, ethnicity, and injection parameters. Clinical characteristics such as substance use and years since most significant trauma or PTSD index trauma are outlined in Table 1 with study schematic shown in Figure 1. As expected, total PTSD severity measured by scan-day PTSD Checklist (PCL) (with diagnosis of PTSD previously confirmed using the Clinician Administered PTSD Scale) was significantly higher in the PTSD group versus the TC group $(P<0.001)$. In the PTSD group, 10 participants had comorbid major depressive disorder (MDD), and 10 were taking psychotropic medications at the time of screening ( 7 for serotonin reuptake inhibitor/antagonist, 2 for norepinephrine-dopamine reuptake inhibitor, 3 for $\mathrm{GABA}_{\mathrm{A}}$ agonist, and 1 for $\alpha_{2}$ agonist).

Imaging group differences in 11ß-HSD1 in a prefrontal-limbic circuit. $\left[{ }^{18} \mathrm{~F}\right] \mathrm{AS} 2471907$ is a radiotracer specific for $11 \beta-\operatorname{HSD} 1(19,35)$ and was shown to be suitable for imaging of 11ß-HSD1 in healthy individuals (20). Regional and composite $\left.{ }^{[18} \mathrm{F}\right] \mathrm{AS} 2471907 V_{\mathrm{T}}$ values were not normally distributed and were log-transformed (log-base 10) in all analyses. Availability of 11ß-HSD1 in PTSD group versus TC group was assessed quantitatively using $\left[{ }^{18} \mathrm{~F}\right]$ AS2471907 volume of distribution or $V_{\mathrm{T}}$. Given preclinical studies showing greater cortical and limbic 11ß-HSD1 expression in association with HPA axis suppression (22) and fear memory potentiation (27), the primary hypothesis proposed that the PTSD group would have higher 11ß-HSD1 availability. This group difference was analyzed across a prefrontal-limbic circuit of a priori regions-amygdala, anterior cingulate cortex (ACC), hippocampus, ventromedial prefrontal cortex (vmPFC) - heavily implicated in the neuroendocrine dysregulation underlying PTSD (36, 37). There was a significant overall main effect in the PTSD group versus the TC group in the identified prefrontal-limbic circuit $(\beta$ $=1.16, P=0.0057$; Figure 2A), indicating that $11 \beta$-HSD1 availability was $16 \%$ higher overall in the PTSD group compared with the TC group across the prefrontal-limbic circuit. Representative images from an individual with PTSD and a sex- and age-matched TC are shown in Figure 2B. Post hoc linear comparisons in each ROI revealed a trending effect of group on 11 $\beta$-HSD1 availability in amygdala $(\beta=1.23, P=0.096)$. Mean $\left[{ }^{18} \mathrm{~F}\right] \mathrm{AS} 2471907 V_{\mathrm{T}}$ values were higher in the PTSD group, by $29 \%$ in amygdala, $25 \%$ in ACC, $9 \%$ in hippocampus, and 17\% in vmPFC. Exploratory analysis revealed no significant group difference across ROIs outside the prefrontal-limbic circuit (Supplemental Figure 1; supplemental material available online with this article; https://doi.org/10.1172/ JCI150452DS1). $\left[{ }^{18} \mathrm{~F}\right] \mathrm{AS} 2471907$ parent fractions in arterial plas-
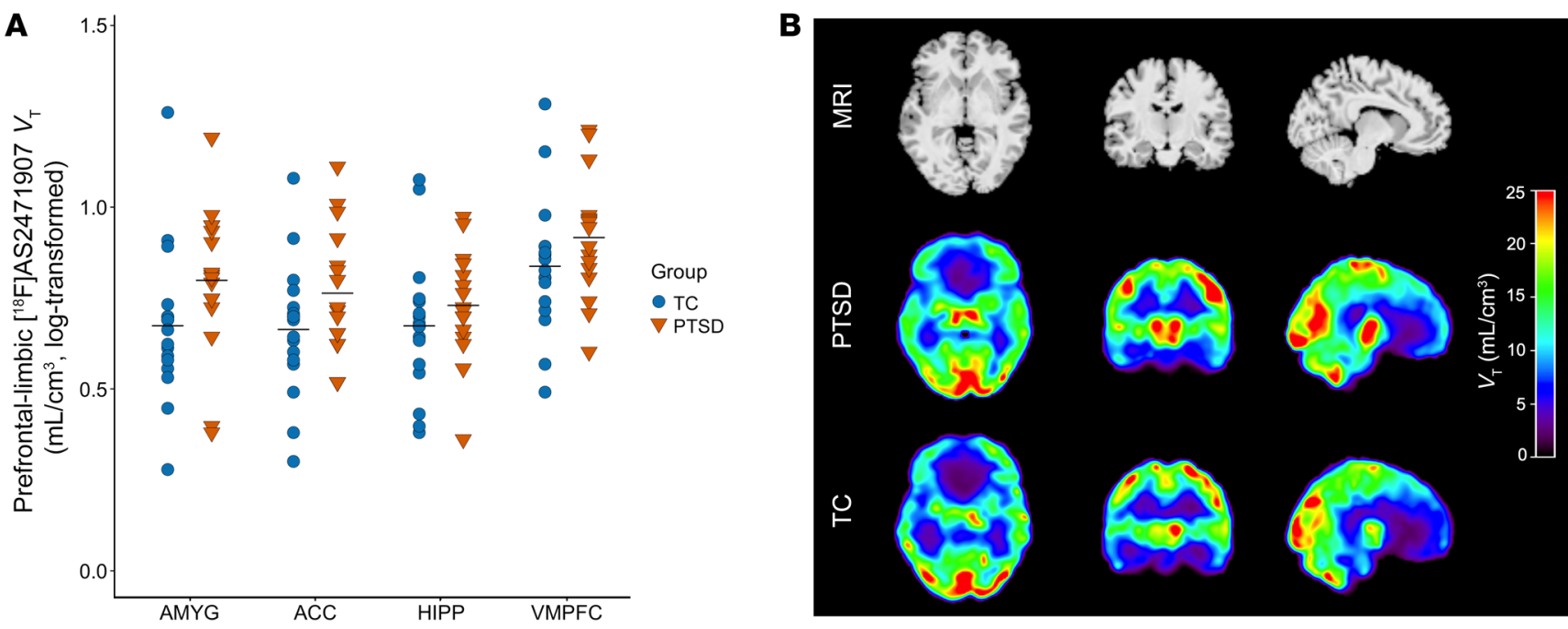

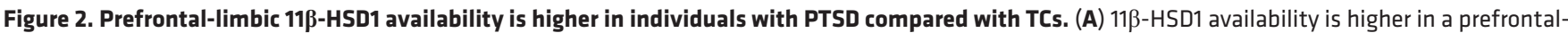
limbic circuit in individuals with PTSD (shown as orange triangles) compared with TCs (shown as blue circles) (group: $\beta=1.16, P=0.0057$ ). Croup differences were assessed using a univariate ANOVA with group as a between-subject factor and ROI as a within-subject factor. Displayed are log-transformed $\left[{ }^{18} \mathrm{~F}\right]$ AS2471907 $V_{T}$ values and horizontal bars indicate group-wise mean. AMYG, amygdala; ACC, anterior cingulate cortex; HIPP, hippocampus; VMPFC, ventromedial prefrontal cortex. (B) Parametric images of absolute $\left[{ }^{18} \mathrm{~F}\right] A S 2471907 V_{T}$ values are shown in representative age- and sex-matched individuals from the PTSD and TC groups. 


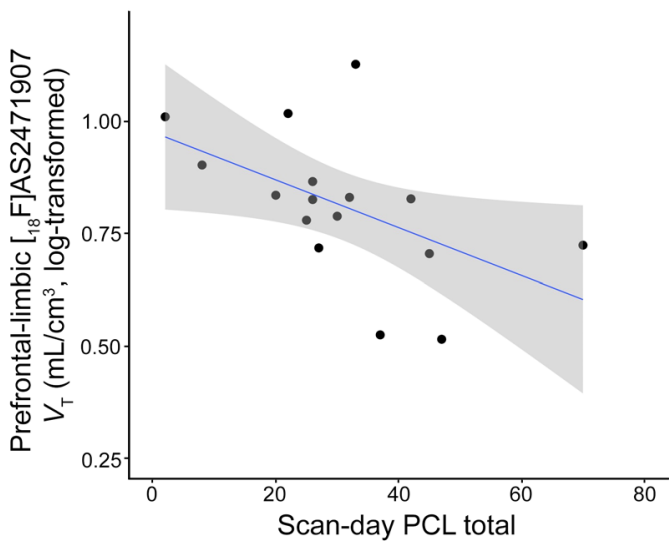

Figure 3. Lower prefrontal-limbic 11/-HSD1 availability is associated with greater PTSD severity. Lower composite prefrontal-limbic [18 F]AS2472907 $V_{\mathrm{T}}$ values were significantly associated with greater overall PTSD severity, assessed as the scan-day total on the PCL $\left(n=16, R^{2}=0.27, P=0.038\right)$. Coefficient of determination was assessed using linear regression. Displayed are log-transformed $\left[{ }^{18} \mathrm{~F}\right] A S 2471907 \mathrm{~V}_{\mathrm{T}}$ values.

ma were comparable across groups (e.g., 150 minutes after injection, $81 \% \pm 7 \%$ for PTSD versus $76 \% \pm 13 \%$ for TC).

Relationship of $11 \beta-H S D 1$ to PTSD symptomatology. To investigate possible relationships between brain cortisol regulation and PTSD symptom heterogeneity, 11 $\beta$-HSD1 availability was assessed in relation to overall PTSD severity and PTSD symptom dimensions. In the PTSD group, greater overall PTSD severity (scan-day PCL total) was significantly associated with lower composite prefrontal-limbic 11 $\beta$-HSD1 availability $\left(R^{2}=0.27, P=0.038\right.$; Figure 3). As expected, this association was only observed in the PTSD group, given the limited range of total symptom severity in the TC group that showed no association with composite prefrontallimbic $11 \beta$-HSD1 availability $(P=0.74)$. There also was no association of total symptom severity with composite prefrontal-limbic $11 \beta$-HSD1 availability across the whole group $(P=0.97)$. Heterogeneity in HPA axis measures in relation to threat $(5,10)$ and loss symptoms (11) led to investigation of 11ß-HSD1 availability in relation to these symptom dimensions derived from a 7-factor model of PTSD (38). Threat was a hybrid of 2 factors, reexperiencing and anxious arousal, and loss reflected the factor comprised of loss of interest, emotional detachment, and restricted affect. Lower prefrontal-limbic 11ß-HSD1 availability was significantly associated with higher scan-day threat symptoms $\left(R^{2}=0.35, P=0.015\right.$; Figure $4 \mathrm{~A})$, as well as higher scan-day loss symptoms $\left(R^{2}=0.39, P=\right.$ 0.010; Figure 4B) in the PTSD group. Post hoc exploratory analyses showed no significant associations between 11ß-HSD1 and any of the remaining factors of the 7-factor model (i.e., avoidance, negative affect, externalizing behaviors, or dysphoric arousal).

Comparing measures of central versus peripheral cortisol regulation. An important goal of this investigation was to examine relationships between a putative marker of brain cortisol regulation, $11 \beta-H S D 1$, and conventional peripheral measures of HPA axis regulation. Exploration of associations with peripheral cortisol showed no significant relationships between composite prefrontal-limbic 113-HSD1 availability and average of plasma cortisol over the 90-min collection period during the PET scan $\left(R^{2}=\right.$ 0.026, $P=0.37$; Supplemental Figure 2). Importantly, the time of scan and accompanying plasma cortisol measurement were carefully controlled to start between 11:00 am and 2:00 pm for each participant, in order to avoid possible diurnal variability in plasma cortisol between participants. There was no evidence of a group by cortisol interaction effect on $11 \beta$-HSD1 availability. Average peripheral cortisol was not correlated to 11ß-HSD1 availability across the whole brain either $\left(R^{2}=0.019, P=0.44\right)$. Average peripheral cortisol was not significantly different between groups $(P=0.65)$ and not associated with scan-day overall symptom severity, threat, or loss symptoms (all $R^{2}<0.18$, all $P>0.11$ ).

Exploration of brain glucocorticoid and neuroimmune systems. Recent findings of lower levels of the microglial marker TSPO (33), and sex-specific lower brain expression of immune-related genes (32) in PTSD have challenged assumptions that the widely observed peripheral inflammation in PTSD would be associated with neuroinflammation. In a subset of 10 individuals in the PTSD
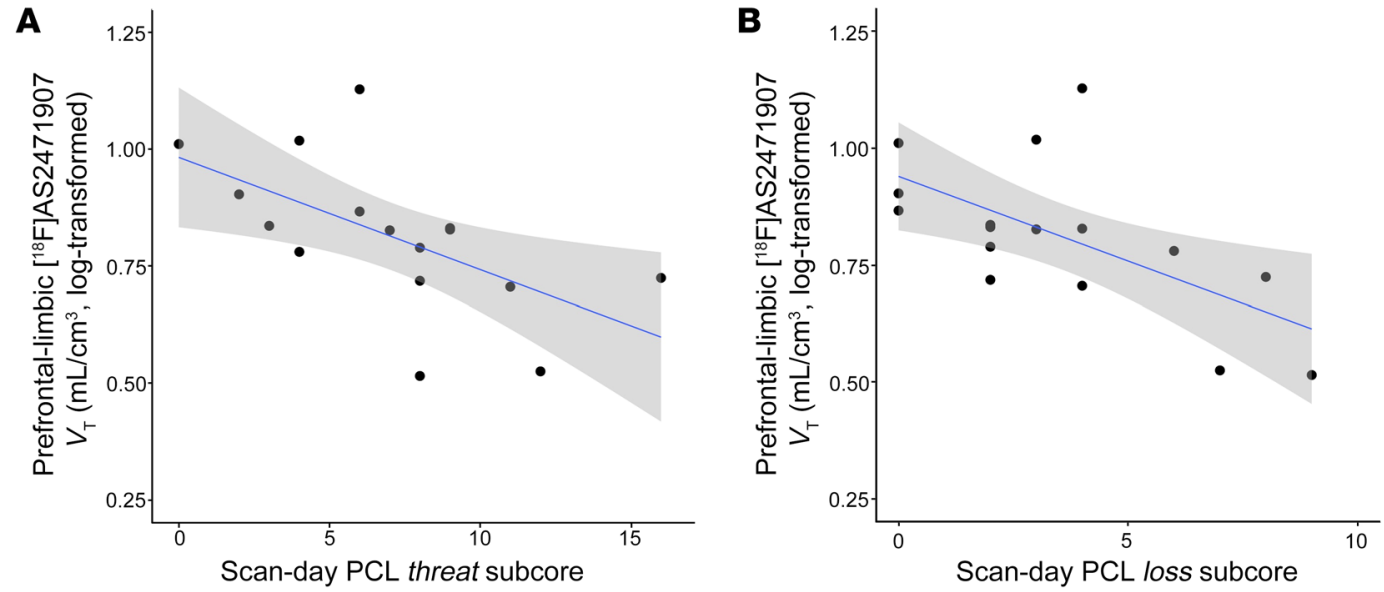

Figure 4. Prefrontal-limbic 11ק-HSD1 availability is associated with PTSD threat and loss symptoms. Lower composite prefrontal-limbic [18 $\mathrm{F}$ AS2472907 $V_{T}$ values were significantly associated with $(\mathbf{A})$ greater threat $\left(R^{2}=0.35, P=0.015\right)$ and $(\mathbf{B})$ greater loss $\left(R^{2}=0.39, P=0.010\right)$ severity. No significant relationships were observed between prefrontal-limbic $\left[{ }^{18} \mathrm{~F}\right] A S 2472907 V_{T}$ and the other factors of the 7-factor model. Coefficient of determination was assessed using linear regression. Displayed are log-transformed $\left[{ }^{18} \mathrm{~F}\right] A S 2471907 V_{T}$ values. 

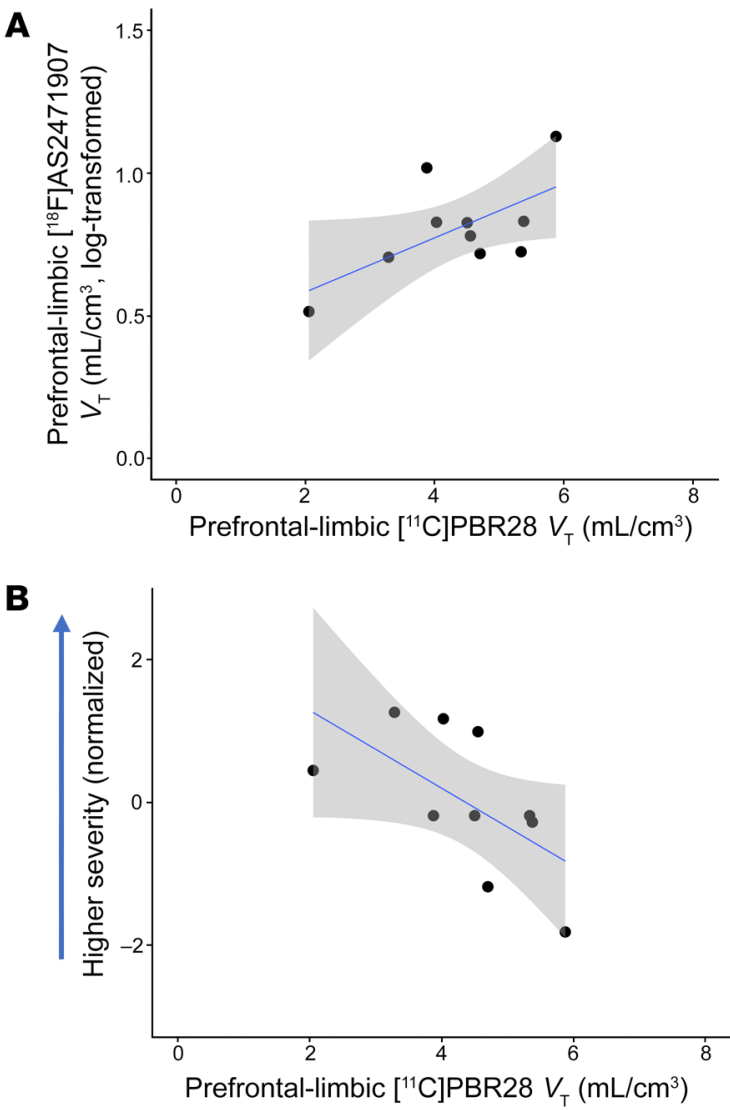

group (rs6971 genotype: $8 \mathrm{HAB}, 2 \mathrm{MAB}$; ref. 39), associations between 11ß-HSD1 and TSPO availability were explored to shed light on a possible immune-modulatory interaction of brain glucocorticoid and microglial signaling that may explain the apparent immune suppression in PTSD. Exploratory analysis revealed a significant positive association between prefrontal-limbic 11 $\beta$-HSD1 and TSPO availability $\left(R^{2}=0.72, P=0.012\right.$; Figure $\left.5 \mathrm{~A}\right)$, and a significant main effect of higher $11 \beta$-HSD1 availability being associated with higher TSPO availability $(\beta=4.40, P=0.039)$ after adjusting for TSPO binding status. Though not reaching statistical significance in this smaller subgroup, the same direction of associations was observed between greater PTSD severity and lower availability of TSPO $\left(R^{2}=0.65, P=0.025\right.$; main effect of severity: $\beta$ $=-0.058, P=0.092)$ and lower availability of $11 \beta-\operatorname{HSD} 1\left(R^{2}=0.20\right.$, $P=0.20)$, as the statistically significant associations observed in the overall groups from each respective study (Figure 5B).

\section{Discussion}

In this study we used a novel PET radiotracer to examine $11 \beta$-HSD1, a putative marker of brain cortisol, in vivo in individuals with PTSD. Overall, 11 $\beta$-HSD1 availability in a prefrontal-limbic circuit was higher in the PTSD group versus the TC group. Interestingly, we observed that higher prefrontal-limbic 11 $\beta$-HSD1 availability in the PTSD group was related to lower overall severity of PTSD symptoms. Additionally, higher 11ß-HSD1 availability was associated with lower threat and loss symptoms, but not with other PTSD dimensions. Thus, it is possible that rather than driving worse symptomatology and brain cortisol dysfunction, the
Figure 5. 11ß-HSD1 availability is associated with availability of TSPO, a microglial marker, and both predict lower symptoms. (A) Higher $\left[{ }^{11} \mathrm{C}\right]$ PBR28 $V_{T}$ is significantly associated with higher $\left[{ }^{18} \mathrm{~F}\right] \mathrm{AS} 241907 \mathrm{~V}_{\mathrm{T}}$ (log-transformed) in the same participants $(n=10)$ in a composite prefrontal-limbic region $\left(R^{2}=0.72, P=0.012 ; \beta=4.40, P=0.039\right.$, adjusting for rs6971 genotype). (B) In this subgroup, higher prefrontal-limbic [" $C]$ PBR28 $V_{\mathrm{T}}$ was associated at a weak trend level with lower PTSD severity $\left(R^{2}=\right.$ $0.65, P=0.025 ; \beta=-0.058, P=0.092$, adjusting for $r s 6971$ genotype), and the same direction of association, though nonsignificant, was observed for [18F]AS241907 $V_{T}$ and PTSD severity $\left(R^{2}=0.20, P=0.20\right)$. Coefficient of determination and standardized coefficients were assessed using linear regression. Displayed are log-transformed $\left[{ }^{18} \mathrm{~F}\right] A S 2471907 V_{T}$ values and genotype-adjusted ["1'C]PBR28 $V_{T}$ values. higher 11ß-HSD1 levels observed in the PTSD group may represent an adaptive compensation associated with less severe PTSD symptomatology. Importantly, the lack of an association between $11 \beta$-HSD1 and basal peripheral cortisol levels raises the possibility that $11 \beta$-HSD1 availability may predict symptom dimensions independently of peripheral cortisol. Preliminarily, a positive association of 11ß-HSD1 availability with TSPO availability within a PTSD subgroup suggests 2 possible molecular markers of neural immune-stress network interactions in PTSD. Thus, 11 $\beta$-HSD1 appears to be a specific marker of brain glucocorticoid regulation in relation to PTSD pathophysiology, which may also have important interactions with the neuroimmune system.

It would appear that the higher prefrontal-limbic 11 $\beta$-HSD1 availability observed in the PTSD group is driven by individuals with lower PTSD severity, rather than those with higher severity. The association of higher 11ß-HSD1 availability with lower overall PTSD severity and specifically with threat symptoms may reflect a compensatory role for brain glucocorticoid signaling in the fear memory-related pathophysiology of PTSD. Though preclinical experiments inhibiting 11ß-HSD1 showed impaired fear memory consolidation (27), several other preclinical experiments employing direct manipulations of systemic and brain corticosteroid concentrations implicated intact brain glucocorticoid signaling in enhanced fear extinction (40-44). Moreover, preliminary evidence from clinical trials suggests that administration of oral corticosteroids can decrease reexperiencing symptoms (45), improve fear extinction deficits in PTSD (46), and enhance the efficacy of exposure therapy (47), thus supporting a role for 
increased brain cortisol signaling in the augmentation of extinction-based therapeutic interventions.

The observation that lower prefrontal-limbic 11ß-HSD1 availability was also associated with greater loss symptoms may represent either a specific association with this symptom dimension or the tendency of threat and loss symptoms to be correlated with each other and with overall PTSD severity. Such relationships between threat and loss dimensions have been reported in functional neuroimaging studies in which decreased prefrontal cortical suppression of amygdala was associated with greater anhedonia (48) and greater attempts to decrease negative affective response to fearful negative imagery (49), which were, in turn, associated with HPA axis dysregulations. The prevalence of both greater threat and loss in individuals with lower 11ß-HSD1 availability may thus reflect an inability of emotional numbing to contain negative affect related to intrusive memories and hyperarousal, thus contributing to higher overall PTSD severity driven by both these symptom dimensions.

Though we had hypothesized a role for higher 11 $\beta-H S D 1$ as a mediator of the chronically suppressed HPA axis activity reported in PTSD, we did not observe associations between higher $11 \beta$-HSD1 and lower HPA axis activity, as assessed by our measure of 90-minute plasma cortisol. There could be a few reasons why analyses in the present study did not detect a possible underlying role of $11 \beta$-HSD1, a central cortisol-generating enzyme, as a link between peripheral cortisol and PTSD symptomatology. Notably, the association between lower peripheral cortisol and PTSD may itself be influenced by factors such as degree of PTSD symptom severity or symptom dimension, which could potentially be obscured when measuring peripheral cortisol across individuals grouped under a singular diagnosis of PTSD (4). It remains possible that some of this symptom heterogeneity can be attributed directly to peripheral cortisol and its effects on more somatic PTSD symptoms. Previous work has suggested that lower peripheral cortisol in PTSD resulted in a failure to contain hyperactivation of the sympathetic nervous response, driven by traumatic memories, and thus contributing to somatic symptoms and behaviors such as elevated heart rate, exaggerated startle response, or dissociation $(8,50,51)$. Additionally, the type of peripheral cortisol assay has been shown to greatly influence the heterogeneity of cortisol findings in PTSD (4). Also, though we carefully controlled the time of cortisol assay, different associations with PTSD have been revealed for morning and afternoon cortisol levels (4) which may not be represented in the 11:00 am to 2:00 pm collection window. Accordingly, the present findings do not preclude the possibility of $11 \beta$-HSD1 as a mediator of HPA axis hypersuppression measured by a different assay or in a different PTSD sample, in which such HPA axis dysregulation and associated symptomatology are present to a greater degree. Last, because $11 \beta-H S D 1$ availability in vivo in humans is not necessarily the equivalent of its enzyme activity and thus of specific levels of brain cortisol, it is possible that a true relationship between central cortisol and peripheral cortisol in PTSD is not captured.

Based on the present findings, we propose a possible model of brain glucocorticoid dysregulation in PTSD in which higher brain levels of 11 $\beta$-HSD1 may develop as a compensatory neuroadaptation. Preclinical studies demonstrate that both exogenous cortico- steroids and psychological stressors produce modest increases in peripheral cortisol subject to negative feedback regulation, while simultaneously upregulating 11 $\beta$-HSD1 expression and activity to produce increases in brain cortisol via positive feed-forward potentiation $(16,52,53)$. The increased brain cortisol signaling during reexposure to traumatic reminders may thereby facilitate formation of fear extinction memories in place of the original fear memory. Brain cortisol-mediated facilitation of fear extinction could contribute to the observed lower levels of threat symptoms, such as intrusive recollections and physiological and emotional reactivity to trauma reminders, by attenuating the cycle of reactivation and reconsolidation of the original fear memory with each instance of reexperiencing. The brain glucocorticoid signaling may also oppose noradrenergic signaling $(30,54,55)$, elevations of which are known to drive threat symptoms of intrusive memories and hyperarousal $(50,56)$. Increased expression of the brain cortisol-producing enzyme, 11 $\beta$-HSD1, could be a compensation to overcome GR resistance and increased FKBP5-GR interactions, which were recently identified in PTSD and rodent fearconditioning models (23) and in contrast with previous findings of heightened GR sensitivity in PTSD (13).

Complementing these findings, we also observed that 11 $\beta$-HSD1 and TSPO availability, a biomarker of microglia, were positively correlated, and each was negatively associated with symptom severity, in the subgroup of individuals who participated in both studies. Indeed, there is emerging support in preclinical PTSD studies for adaptive roles of both brain cortisol signaling and brain immune responses. Barrientos and colleagues found that higher hippocampal 11ß-HSD1 levels in rats were associated with higher levels of markers of both glucocorticoid signaling and microglial activation, but also with reduction of conditioned fear responding following an $E$. coli immune challenge, an effect that was blocked by mifepristone, a glucocorticoid antagonist (57). Thus, one interpretation of our data in conjunction with these preclinical findings is that stress-induced greater brain glucocorticoid signaling may sensitize microglia, allowing for a neuroprotective neuroimmune response that results in less severe PTSD symptoms. These findings collectively challenge previous hypotheses of the deleterious effects of both excessive brain glucocorticoid and brain immune signaling in PTSD and point us toward joint examination of deficiency in these systems in the pathophysiology of PTSD (58).

A few limitations of this study should be noted. First, $\left.{ }^{18} \mathrm{~F}\right]$ AS2471907 $V_{\mathrm{T}}$ reflects the number of 11ß-HSD1 sites available to bind $\left[{ }^{18} \mathrm{~F}\right] \mathrm{AS} 2471907$, which is interpreted as a proxy for $11 \beta-\mathrm{HSD} 1$ enzyme density. This interpretation is supported by the associations between regional patterns of $11 \beta$-HSD1 availability and 11ß-HSD1 mRNA expression (Allen Human Brain Atlas) observed in our previous study (20). As enzyme density is not necessarily equivalent to enzyme activity, a potential indirect relationship of $\left[{ }^{18} \mathrm{~F}\right] \mathrm{AS} 2471907 V_{\mathrm{T}}$ and $11 \beta$-HSD1 enzyme activity could partly explain why an association between 11/-HSD1 availability and peripheral cortisol was not observed. However, evidence from postmortem studies of aging men suggests a correlation between $11 \beta$-HSD1 mRNA expression and ex vivo 11ß-HSD1 activity in hippocampus and cerebellum (24). Furthermore, the approximately 4 -fold greater 11 $\beta$-HSD1 enzyme activity of cerebellar tissue 
compared with hippocampal tissue paralleled the approximately 4 -fold greater 11 $\beta$-HSD1 availability in cerebellum compared with hippocampus seen here and reported previously $(20,24)$. Nonetheless, full extrapolation from availability to activity will require ongoing studies to directly assay in vitro $\left.{ }^{18} \mathrm{~F}\right] \mathrm{AS} 2471907$ binding in relation to $11 \beta-H S D 1-m e d i a t e d$ cortisol production. Second, the present study was not powered to analyze possible effects of sex, but we have matched the PTSD and TC groups on sex. Third, we did not exclude for comorbid nicotine and cannabis use, given the prevalence of these disorders in the PTSD population (1), and instead the TC group is matched for these conditions. We also did not exclude comorbid MDD or use of certain psychotropic medications to allow for a representative PTSD sample, and though the study was not powered to analyze the effect of these factors, we feel it is a strength that our sample is more generalizable. Fourth, given the known heterogeneity in the finding of hypocortisolism in PTSD that is dependent on type and time of cortisol assay (4), the observed lack of association between peripheral cortisol and $11 \beta$-HSD1 availability in the present study could be followed up with additional evaluation of HPA axis function such as daily cortisol output or characterization of diurnal cortisol rhythm and cortisol response to dexamethasone challenge.

Overall, the association of greater 11ß-HSD1 levels with lower PTSD symptom severity observed in our study provides a novel finding about the brain cortisol system. Moreover, these data generate hypotheses of a neuroadaptive role for brain cortisol signaling in stress-related pathology associated with PTSD, shedding light on a potential mechanism underlying the therapeutic response to cortisol administration in PTSD (59). This first step toward greater understanding of the brain cortisol system in vivo in PTSD paves the way for future interrogations of this system-longitudinal studies at multiple time points following index trauma, pharmacological challenges to alter 11ß-HSD1 levels, and characterization of functional HPA axis and neuroimmune responses in relation to 11 $\beta$-HSD1. Undoubtedly, there is an emerging role for $11 \beta$-HSD1 as an important molecular player in the glucocorticoid system in PTSD.

\section{Methods}

Participant characteristics. Sixteen individuals with PTSD (8 female, 8 male) and 17 healthy control individuals with trauma exposure (TCs) (8 female, 9 male) participated in the study. Psychiatric screening was conducted by clinically trained staff with supervision from a licensed clinical psychologist. The Structured Clinical Interview for the DSM-5 (SCID-5) and the Clinician Administered PTSD Scale for the DSM-5 (CAPS-5) were used to determine current diagnosis of PTSD in the PTSD group, and no current or past diagnosis of PTSD in the TC group. In all subjects, the SCID-5 was used to rule out exclusionary diagnoses (e.g., schizophrenia, bipolar disorder). In the PTSD group it was used to determine comorbidity of disorders with high prevalence in the general PTSD population including MDD (60). Similarly, due to high prevalence in individuals with PTSD, nicotine, cannabis, and alcohol use were not exclusionary $(1,61,62)$. Use of other illicit psychoactive substances based on urine drug testing was exclusionary. Individuals currently taking prescribed psychotropic medications other than monoamine (serotonin, norepinephrine, and dopamine) reuptake inhibitors, serotonin antagonists, and $\alpha_{2}$ - and GABA-receptor agonists were not eligible to participate. The study physician oversaw medical screening, including EKG, bloodwork, history, and physical exam, to rule out significant medical illnesses. Current use of steroidal and nonsteroidal antiinflammatory medications was exclusionary.

Trauma history and PTSD severity assessment. History of at least one traumatic event was assessed in all participants by screening with the SCID Trauma and Stress-Related Disorders screen (DSM-5). History of exposures to particular types of trauma as listed in Table 1 was assessed by a combination of participant report during clinician interview, self-report on the Life Events Checklist, a well-validated 17-item tool for assessing trauma history (63), and the Childhood Trauma Questionnaire Short Form (CTQ), a well-validated 28-item measure for assessing history of traumatic events before 18 years of age (64). Total PTSD severity and threat and loss subscores were assessed on the PET scan day by self-report using the PCL for the DSM-5.

Hormone assessments. Peripheral cortisol was measured for all participants on the PET scan day in plasma samples drawn at 0 (immediately prior to), 30, 60, and 90 minutes relative to radiotracer injection (Corti-Cote Cortisol Solid Phase Component System, MP Biomedicals LLC). Plasma cortisol measurements started between 11:00 am and 2:00 pm across all participants in accordance with tightly controlled timing of the radiotracer injection.

Imaging acquisition. Participants were given an intravenous bolus injection of $93 \pm 15 \mathrm{MBq}$ of $\left[{ }^{18} \mathrm{~F}\right] \mathrm{AS} 2471097$, which was synthesized as previously described (19), and imaged for up to $150(n=6), 180$ $(n=24)$, or $240(n=3)$ minutes after injection on a High-Resolution Research Tomograph (HRRT; Siemens, Medical Solutions) with a 30-minute break starting at $90(n=6)$ or $120(n=27)$ minutes. All participants were scanned starting between 11:00 am and 2:00 pm, to reduce possible diurnal variability in 11ß-HSD1 availability in association with well-established diurnal cortisol rhythms (65). A structural image for anatomical coregistration was acquired with a high-resolution T1-weighted MRI with a sagittal gradient-echo (MPRAGE) sequence (Siemens 3.0T Prisma Fit; 176 sagittal slices, thickness = $1 \mathrm{~mm}, \mathrm{TR}=2530 \mathrm{~ms}, \mathrm{TE}=2.26 \mathrm{~ms}$, flip angle $=7^{\circ}, \mathrm{FoV}=256 \mathrm{~mm}$, matrix size $=256 \times 256$ ).

Arterial input function measurement. An arterial catheter was placed in the radial artery contralateral to the injection site for $\left[{ }^{18} \mathrm{~F}\right]$ AS2471907, for arterial sampling throughout the duration of the scan. Radioactivity was measured in manual samples collected every $16 \mathrm{sec}-$ onds from 0 to 2.5 minutes, then at $2.75,3,5,10,20$, and 30 minutes, and every 15 minutes thereafter until 120 minutes or 150 minutes, then every 30 minutes for scans longer than 150 minutes. Fraction of unmetabolized $\left[{ }^{18} \mathrm{~F}\right] \mathrm{AS} 2471907$ was measured using high-performance liquid chromatography (HPLC) as previously described (66) in plasma samples taken at $0,10,30,60,90,120$, and 150 minutes, or every 60 minutes after the 120 minute time point for scans longer than 150 minutes. A metabolite-corrected arterial input function was calculated as the product of the plasma radioactivity and unmetabolized $\left[{ }^{18} \mathrm{~F}\right] \mathrm{AS} 2471907$ fraction, with radioactivity measured with a crosscalibrated well counter (1480 Wizard, Perkin-Elmer).

PET imaging analysis. PET data were collected in list-mode and reconstructed using MOLAR (67) with correction for attenuation, scatter, randoms, deadtime, and subject motion recorded by Vicra (Polaris Vicra Optical Tracking System, NDI Systems). A 7-minute transmission scan was done prior to radiotracer injection and immediately following completion of full PET data acquisition, with the 
latter transmission scan allowing attenuation correction for potential large shifts in participant position in the scanner following the 30-minute break. A 0 to 10 minute summed PET image was linearly coregistered to each individual's T1-weighted structural MR image. Nonlinear transformation from Montreal Neurological Institute (MNI) atlas space to individual MR space and an anatomic automatic labeling (AAL) template were used to generate time activity curves in the following ROIs: frontal, occipital, temporal, parietal, ventromedial prefrontal, orbitofrontal, and anterior cingulate cortices, amygdala, hippocampus, insula, caudate, putamen, cerebellum, thalamus, and in a whole brain ROI. Based on previous characterization of optimal modeling methods (20), the multilinear analysis (MA1) modeling approach with $\mathrm{t}^{*}=30$ minutes was used to estimate regional $\left[{ }^{18} \mathrm{~F}\right] \mathrm{AS} 2471907$ volume of distribution or $V_{\mathrm{T}}$, defined as the ratio of radioactivity in tissue to plasma at equilibrium (68). To consider participant comfort in ongoing studies, analyses of truncated data revealed that 150 minutes of scan acquisition yielded $V_{\mathrm{T}}$ values in good agreement (within 5\%) with values of $V_{\mathrm{T}}$ estimated from the previously validated 180 -minute scan length, and in good agreement (within 10\%) with values of $V_{\mathrm{T}}$ estimated from 240 minutes of data in 8 individuals (20). Therefore, $V_{\mathrm{T}}$ values were estimated with the first 150 minutes of data across all participants for consistency. Voxelwise parametric images were generated using $1 \mathrm{~T}$ compartment model and smoothing with a $7 \mathrm{~mm}$ Gaussian kernel, with this method being chosen for reasons previously described (20).

$\left[{ }^{11} \mathrm{C}\right]$ PBR28 PET data comparison. In a subgroup of individuals with PTSD who had also completed $\left[{ }^{11} \mathrm{C}\right] \mathrm{PBR} 28$ scans $(n=9$ scanned $36 \pm$ 26 days apart; $n=1$ scanned 2.5 years apart), $\left.{ }^{18} \mathrm{~F}\right] \mathrm{AS} 2471907 V_{\mathrm{T}}$ was examined in relation to $\left.{ }^{11} \mathrm{C}\right] \mathrm{PBR} 28 V_{\mathrm{T}}$ in a prefrontal-limbic region. $\left[{ }^{11} \mathrm{C}\right]$ PBR28 imaging acquisition, arterial input function measurement, and imaging analysis used to obtain $\left[{ }^{11} \mathrm{C}\right] \mathrm{PBR} 28 V_{\mathrm{T}}$ values in this subgroup were reported previously (33). Within this subgroup, total PTSD severity was obtained from total CAPS scores obtained during participation in the $\left[{ }^{11} \mathrm{C}\right] \mathrm{PBR} 28$ study, as previously reported (33). Exploratory linear regression analyses assessed possible associations of $\left[{ }^{18} \mathrm{~F}\right] \mathrm{AS} 2471907 V_{\mathrm{T}}$ in a composite prefrontal-limbic region with $\left[{ }^{11} \mathrm{C}\right]$ PBR2 $8 V_{\mathrm{T}}$ values in a composite region formed of the same ROIs (amygdala, ACC, hippocampus, and vmPFC). These analyses were adjusted for $r s 6971$ genotype (70) which confers differential binding affinity of $\left[{ }^{11} \mathrm{C}\right]$ PBR28 for TSPO (39). Relationships of composite $\left[{ }^{18} \mathrm{~F}\right]$ AS2471907 $V_{\mathrm{T}}$ and $\left[{ }^{11} \mathrm{C}\right] \mathrm{PBR} 28 V_{\mathrm{T}}$ with overall PTSD severity scores, measured using PCL and CAPS, respectively, were also explored. Overall PTSD severity scores were normalized to the same scale for visualization purposes.

Statistics. Demographic characteristics and injection parameters were compared between the PTSD group and the TC group using Student's $t$ tests, Mann-Whitney $U$ tests, or $\chi^{2}$ tests, where appropriate. Normality of dependent variables in all linear modeling analyses was determined using Shapiro-Wilk tests and examination of QQ distributions with log-transformations as necessary to stabilize variance and satisfy modeling assumptions. Regional and composite $\left.{ }^{[18} \mathrm{F}\right] \mathrm{AS} 2471907 V_{\mathrm{T}}$ values that were not normally distributed were log-transformed (log-base 10) to produce normal distributions across participants and within PTSD and TC groups.

The main effect of the PTSD group versus the TC group on prefrontal-limbic 11ß-HSD1 availability was examined using a univariate ANOVA, with log-transformed $\left[{ }^{18} \mathrm{~F}\right] \mathrm{AS} 2471907 V_{\mathrm{T}}$ as the dependent variable, PTSD group versus TC group as a between-subject factor, and ROI as a within-subject factor for the following a priori ROIs comprising a prefrontal-limbic circuit: amygdala, anterior cingulate cortex (ACC), hippocampus, and ventromedial prefrontal cortex (vmPFC). This main effect of group was tested with appropriate post hoc contrasts for the PTSD group versus TC group within each a priori ROI and also in exploratory analyses in each of the remaining ROIs. Unlike in Bhatt et al. (69), in which insula was included in a prefrontal-limbic circuit based on previous associations of insular TSPO availability with loss symptoms, no such association of insular 11ß-HSD1 expression or density with preclinical threat or loss behaviors has been observed to our knowledge. Thus, in the present study, insula was not included in the prefrontal-limbic circuit. Standardized coefficients ( $\beta$ ), reported for the main effect of the group in these analyses, were reported as multiplicative coefficients of non-log-transformed $V_{\mathrm{T}}$ values (i.e., a $\beta$ of 1.1 corresponds to a $10 \%$ difference in mean $V_{\mathrm{T}}$ values).

A general linear modeling approach was used to compare total PTSD severity and severity of threat and loss symptoms to log-transformed $\left[{ }^{18} \mathrm{~F}\right] \mathrm{AS} 2471907 V_{\mathrm{T}}$ values in a composite average ROI of the a priori prefrontal-limbic ROIs including the amygdala, ACC, hippocampus, and vmPFC. Overall PTSD severity was the total score of the scan-day PCL-5. Threat was a summed subscore of the respective items from the scan-day PCL-5 comprising fear memory-related reexperiencing (intrusive memories, disturbing dreams, flashbacks, trauma-related psychological reactivity, trauma-related physiological reactivity) and anxious arousal (hypervigilance and exaggerated startle response) from the 7-factor hybrid model of PTSD (38). Similarly, loss was a sum comprised of loss of interest, emotional detachment, and restricted affect items comprising the anhedonia factor from the 7-factor model. Post hoc exploratory analyses were also conducted for the other factors from the 7-factor hybrid model: avoidance, negative affect, externalizing behaviors, and dysphoric arousal.

Additional exploratory linear regression analyses examined whether $\left.{ }^{[18} \mathrm{F}\right] \mathrm{AS} 2471907 V_{\mathrm{T}}$ in a composite prefrontal-limbic ROI and a whole brain ROI, and PTSD symptom severity measures, were related to plasma cortisol averaged over 90 minutes.

For all statistical analyses, a $P$ value less than 0.05 was considered the threshold for statistical significance.

Study approval. The 33 individuals who participated in this study were recruited from 2016 to present via advertisements in public forums. Written informed consent was obtained from all participants after a complete explanation of study procedures. All screening and investigation procedures were in accordance with Code of Federal Regulations Title 45, Part 46 policy on protection of human subjects in research, and were approved and overseen by the Yale University Institutional Human Investigation Committee and the Yale New Haven Hospital Radiation Safety Committee.

\section{Author contributions}

KPC, SB, SMS, and JHK conceived and designed the study. SB analyzed the PET data with input from ATH, REC, and KPC. SB performed statistical analyses on PET, clinical, and peripheral marker data, with ATH and KPC providing statistical expertise and guidance. AR oversaw recruitment and scanning, with supervision from KPC and SB. DM and GAA provided medical expertise. SN performed radiochemical synthesis and $\mathrm{NN}$ and $\mathrm{YH}$ were responsible for supervising radiochemistry. MK conducted metabolite 
analysis. SB wrote the manuscript in consultation with KPC. All authors edited and contributed comments and intellectual content to the manuscript.

\section{Acknowledgments}

We are grateful for the expertise of the staff at the Yale PET Center for their support of radiochemistry and imaging. Additionally, we thank the National Center for PTSD (West Haven Campus) - the faculty for their experimental insight and the staff for their administrative support. We extend deep gratitude to the individuals who participated in the PET study. Yale Center for Clinical Investigation provided support for the cortisol assays. We are thankful for our funding from the Brain and Behavior Foundation
Independent Investigator Grant (to KPC), National Institute of Mental Health grants F30MH116607 (to SB) and R01MH110674 (to KPC), the Veterans Affairs National Center for PTSD, and the Gustavus and Louise Pfeiffer Foundation Fellowship (to SB). This publication was also made possible by Clinical and Translational Science Awards grant UL1 TR000142 from the National Center for Advancing Translational Science (NCATS), a component of the NIH. Its contents are solely the responsibility of the authors and do not necessarily represent the official view of NIH.

Address correspondence to: Kelly Cosgrove, 2 Church Street, Suite 511, New Haven, Connecticut 06519, USA. Phone: 203.737.6969; Email: kelly.cosgrove@yale.edu.
1. Goldstein RB, et al. The epidemiology of DSM-5 posttraumatic stress disorder in the United States: results from the National Epidemiologic Survey on Alcohol and Related Conditions-III. Soc Psychiatry Psychiatr Epidemiol. 2016;51(8):1137-1148.

2. Kilpatrick DG, et al. National estimates of exposure to traumatic events and PTSD prevalence using DSM-IV and DSM-5 criteria. J Trauma Stress. 2013;26(5):537-547.

3. Meewisse M-L, et al. Cortisol and post-traumatic stress disorder in adults: systematic review and meta-analysis. Br J Psychiatry. 2007;191(5):387-392.

4. Morris MC, et al. Relations among posttraumatic stress disorder, comorbid major depression, and HPA function: a systematic review and meta-analysis. Clin Psychol Rev. 2012;32(4):301-315.

5. Olff M, et al. HPA- and HPT-axis alterations in chronic posttraumatic stress disorder. Psychoneuroendocrinology. 2006;31(10):1220-1230.

6. Steudte-Schmiedgen S, et al. An integrative model linking traumatization, cortisol dysregulation and posttraumatic stress disorder: insight from recent hair cortisol findings. Neurosci Biobehav Rev. 2016;68:124-135.

7. Wingenfeld K, et al. Effect of current and lifetime posttraumatic stress disorder on 24-h urinary catecholamines and cortisol: results from the Mind Your Heart Study. Psychoneuroendocrinology. 2015;52:83-91.

8. Yehuda R. Post-traumatic stress disorder. N Eng J Med. 2002;346(2):108-114.

9. Yehuda R, et al. Cortisol regulation in posttraumatic stress disorder and major depression: a chronobiological analysis. Biol Psychiatry. 1996;40(2):79-88.

10. Witteveen AB, et al. Associations of cortisol with posttraumatic stress symptoms and negative life events: a study of police officers and firefighters. Psychoneuroendocrinology. 2010;35(7):1113-1118.

11. Stoppelbein L, Greening L. A longitudinal study of the role of cortisol in posttraumatic stress disorder symptom clusters. Anxiety Stress Coping. 2015;28(1):17-30.

12. Klaassens ER, et al. Adulthood trauma and HPA-axis functioning in healthy subjects and PTSD patients: a meta-analysis. Psychoneuroendocrinology. 2012;37(3):317-331.

13. Yehuda R, et al. The cortisol and glucocorticoid receptor response to low dose dexamethasone administration in aging combat veterans and holocaust survivors with and without posttraumatic stress disorder. Biol Psychiatry. 2002;52(5):393-403.

14. Herman JP, et al. Limbic system mechanisms of stress regulation: hypothalamo-pituitary-adrenocortical axis. Prog Neuropsychopharmacol Biol Psychiatry. 2005;29(8):1201-1213.

15. Meijer OC, et al. Cell- and tissue-specific effects of corticosteroids in relation to glucocorticoid resistance: examples from the brain. JEndocrinol. 2003;178(1):13-18.

16. Yau J, et al. Diurnal and stress-induced intrahippocampal corticosterone rise attenuated in 11ß-HSD1-deficient mice: a microdialysis study in young and aged mice. Eur J Neurosci. 2015;41(6):787-792.

17. Chapman K, et al.11ß-hydroxysteroid dehydrogenases: intracellular gate-keepers of tissue glucocorticoid action. Physiol Rev. 2013;93(3):1139-1206.

18. Yau JL, et al. Lack of tissue glucocorticoid reactivation in 11beta -hydroxysteroid dehydrogenase type 1 knockout mice ameliorates age-related learning impairments. Proc Natl Acad Sci U S A. 2001;98(8):4716-4721.

19. Baum E, et al. A novel 18F-labeled radioligand for positron emission tomography imaging of 11 $\beta$-hydroxysteroid dehydrogenase (11 $\beta$ HSD1): Synthesis and preliminary evaluation in nonhuman primates. ACS Chem Neurosci. 2019;10(5):2450-2458.

20. Bhatt S, et al. First in-human PET study and kinetic evaluation of $\left[{ }^{18} \mathrm{~F}\right] \mathrm{AS} 2471907$ for imaging $11 \beta$-hydroxysteroid dehydrogenase type 1 . J Cereb Blood Flow Metab. 2019;40(4):695-704.

21. Bjorn S, et al. Radiolabeled glucocorticoids as molecular probes for imaging brain glucocorticoid receptors by means of positron emission tomography (PET). Mini Rev Med Chem. 2008;8(7):728-739.

22. Harris $\mathrm{HJ}$, et al. Intracellular regeneration of glucocorticoids by $11 \beta$-hydroxysteroid dehydrogenase (11 $\beta$-HSD)-1 plays a key role in regulation of the hypothalamic-pituitary-adrenal axis: analysis of 11/-HSD-1-deficient mice. Endocrinology. 2001;142(1):114-120.

23. Li H, et al. The glucocorticoid receptor-FKBP51 complex contributes to fear conditioning and posttraumatic stress disorder. JClin Invest. 2020;130(2):877-889.

24. Sandeep TC, et al. 11Beta-hydroxysteroid dehydrogenase inhibition improves cognitive function in healthy elderly men and type 2 diabetics. Proc Natl Acad Sci U S A. 2004;101(17):6734-6739.

25. Yau JLW, et al. 11beta-hydroxysteroid dehydrogenase type 1 deficiency prevents memory deficits with aging by switching from glucocorticoid receptor to mineralocorticoid receptor-mediated cognitive control. J Neurosci. 2011;31(11):4188-4193.

26. Yau JLW, et al. Intrahippocampal glucocorticoids generated by 11 $\beta$-HSD1 affect memory in aged mice. Neurobiol Aging. 2015;36(1):334-343.

27. Sarabdjitsingh RA, et al. Inhibiting $11 \beta$-hydroxysteroid dehydrogenase type 1 prevents stress effects on hippocampal synaptic plasticity and impairs contextual fear conditioning. Neuropharmacology. 2014;81:231-236.

28. American Psychological Association. Diagnostic and Statistical Manual of Mental Disorders. American Psychiatric Publishing; 2013.

29. Zobel AW, et al. Cortisol response in the combined dexamethasone/CRH test as predictor of relapse in patients with remitted depression. a prospective study. J Psychiatr Res. 2001;35(2):83-94.

30. Rohleder N, et al. Hypocortisolism and increased glucocorticoid sensitivity of pro-inflammatory cytokine production in Bosnian war refugees with posttraumatic stress disorder. Biol Psychiatry. 2004;55(7):745-751.

31. Gill J, et al. Low cortisol, high DHEA, and high levels of stimulated TNF-alpha, and IL-6 in women with PTSD. J Trauma Stress. 2008;21(6):530-539.

32. Girgenti MJ, et al. Transcriptomic organization of the human brain in post-traumatic stress disorder. Nat Neurosci. 2021;24(1):24-33.

33. Bhatt S, et al. PTSD is associated with neuroimmune suppression: evidence from PET imaging and postmortem transcriptomic studies. Nat Commun. 2020;11(1):2360.

34. Setiawan $\mathrm{E}$, et al. Association of translocator protein total distribution volume with duration of untreated major depressive disorder: a cross-sectional study. Lancet Psychiatry. 2018;5(4):339-347.

35. Li S, Cai Z, Zhang W, Holden D, Baum E, Nabulsi $\mathrm{N}$, et al. Synthesis and evaluation of a novel $18 \mathrm{~F}$-labeled radiotracer for imaging $11 \beta$-HSD1 in the brain. J Nucl Med. 2018;59(supplement 1):548. 
36. Dedovic K, et al. The brain and the stress axis: the neural correlates of cortisol regulation in response to stress. Neuroimage. 2009;47(3):864-871.

37. Shin LM, et al. Amygdala, medial prefrontal cortex, and hippocampal function in PTSD. Ann N Y Acad Sci. 2006;1071(1):67-79.

38. Armour C, et al. Dimensional structure of DSM-5 posttraumatic stress symptoms: support for a hybrid anhedonia and externalizing behaviors model. J Psychiatr Res. 2015;61:106-113.

39. Owen DRJ, et al. Mixed-affinity binding in humans with $18-\mathrm{kDa}$ translocator protein ligands. J Nucl Med. 2011;52(1):24-32.

40. Kaouane N, et al. Glucocorticoids can induce PTSD-like memory impairments in mice. Science. 2012;335(6075):1510-1513.

41. Cai W-H, et al. Postreactivation glucocorticoids impair recall of established fear memory. J Neurosci. 2006;26(37):9560-9566.

42. Yang Y-L, et al. Systemic and intra-amygdala administration of glucocorticoid agonist and antagonist modulate extinction of conditioned fear. Neuropsychopharmacology. 2006;31(5):912-924.

43. Blundell J, et al. Block of glucocorticoid synthesis during re-activation inhibits extinction of an established fear memory. Neurobiol Learn Mem. 2011;95(4):453-460.

44. Barrett D, Gonzalez-Lima F. Behavioral effects of metyrapone on Pavlovian extinction. Neurosci Lett. 2004;371(2):91-96.

45. Aerni A, et al. Low-dose cortisol for symptoms of posttraumatic stress disorder. Am J Psychiatry. 2004;161(8):1488-1490.

46. Michopoulos V, et al. Dexamethasone facilitates fear extinction and safety discrimination in PTSD: A placebo-controlled, double-blind study. Psychoneuroendocrinology. 2017;83:65-71.

47. de Quervain DJF, et al. Glucocorticoids enhance extinction-based psychotherapy. Proc Natl Acad Sci U S A. 2011;108(16):6621-6625.

48. Putnam KM, et al. Neural activity and diurnal variation of cortisol: evidence from brain electrical tomography analysis and relevance to anhedonia. Psychophysiology. 2008;45(6):886-895.
49. Urry HL, et al. Amygdala and ventromedial prefrontal cortex are inversely coupled during regulation of negative affect and predict the diurnal pattern of cortisol secretion among older adults. J Neurosci. 2006;26(16):4415-4425.

50. Nicholson EL, et al. Interaction of noradrenaline and cortisol predicts negative intrusive memories in posttraumatic stress disorder. Neurobiol Learn Mem. 2014;112:204-211.

51. Morgan CA III, et al. Relationships among plasma dehydroepiandrosterone and dehydroepiandrosterone sulfate, cortisol, symptoms of dissociation, and objective performance in humans exposed to underwater navigation stress. Biol Psychiatry. 2009;66(4):334-340.

52. Low SC, et al. Glucocorticoids regulate hippocampal 11 beta-hydroxysteroid dehydrogenase activity and gene expression in vivo in the rat. J Neuroendocrinol. 1994;6(3):285-290.

53. Rajan V, et al. 11 beta-hydroxysteroid dehydrogenase in cultured hippocampal cells reactivates inert 11-dehydrocorticosterone, potentiating neurotoxicity. J Neurosci. 1996;16(1):65-70.

54. Pacak K, et al. Hypercortisolemia inhibits yohimbine-induced release of norepinephrine in the posterolateral hypothalamus of conscious rats. Endocrinology. 1992;131(3):1369-1376.

55. Jeong K-H, et al. Impaired basal and restraintinduced epinephrine secretion in corticotropin-releasing hormone-deficient mice. Endocrinology. 2000;141(3):1142-1150.

56. Geracioti TD, et al. CSF norepinephrine concentrations in posttraumatic stress disorder. Am J Psychiatry. 2001;158(8):1227-1230.

57. Barrientos RM, et al. Greater glucocorticoid receptor activation in hippocampus of aged rats sensitizes microglia. Neurobiol Aging. 2015;36(3):1483-1495.

58. Heim C. Deficiency of inflammatory response to acute trauma exposure as a neuroimmune mechanism driving the development of chronic PTSD: another paradigmatic shift for the conceptualization of stress-related disorders? Am J Psychiatry. 2020;177(1):10-13.

59. de Quervain D, et al. Stress, glucocorticoids and memory: implications for treating fear-related disorders. Nat Rev Neurosci. 2017;18(1):7-19.

60. Smith SM, et al. The association between post-traumatic stress disorder and lifetime DSM-5 psychiatric disorders among veterans: data from the National Epidemiologic Survey on Alcohol and Related Conditions-III (NESARCIII). J Psychiatr Res. 2016;82:16-22.

61. Cougle JR, et al. Posttraumatic stress disorder and cannabis use in a nationally representative sample. Psychol Addict Behav. 2011;25(3):554-558.

62. Gentes EL, et al. Prevalence and correlates of cannabis use in an outpatient VA posttraumatic stress disorder clinic. Psychol Addict Behav. 2016;30(3):415-421.

63. Gray MJ, et al. Psychometric properties of the life events checklist. Assessment. 2004;11(4):330-341.

64. Bernstein DP, et al. Development and validation of a brief screening version of the Childhood Trauma Questionnaire. Child Abuse Negl. 2003;27(2):169-190.

65. Stone AA, et al. Individual differences in the diurnal cycle of salivary free cortisol: a replication of flattened cycles for some individuals. Psychoneuroendocrinology. 2001;26(3):295-306.

66. Hilton J, et al. Column-switching HPLC for the analysis of plasma in PET imaging studies. Nucl Med Biol. 2000;27(6):627-630.

67. Carson RE, et al. Design of a motion-compensation OSEM list-mode algorithm for resolution-recovery reconstruction for the HRRT. Paper presented at: 2003 IEEE Nuclear Science Symposium; October 19-25, 2003; Portland, Oregon, USA. https://doi.org/10.1109/NSSMIC.2003.1352597. Accessed September 2, 2021.

68. Innis RB, et al. Consensus nomenclature for in vivo imaging of reversibly binding radioligands. J Cereb Blood Flow Metab. 2007;27(9):1533-1539.

69. Holmes SE, et al. Elevated translocator protein in anterior cingulate in major depression and a role for inflammation in suicidal thinking: a positron emission tomography study. Biol Psychiatry. 2018;83(1):61-69.

70. Owen DR, et al. An 18-kDa translocator protein (TSPO) polymorphism explains differences in binding affinity of the PET radioligand PBR28. JCereb Blood Flow Metab. 2011;32(1):1-5. 